\title{
Risk factors in bee and Vespula venom allergy: state of the art
}

\author{
Johanna Stoevesandt · Axel Trautmann
}

Received: 19 May 2021 / Accepted: 30 July 2021 / Published online: 15 September 2021

(C) The Author(s) 2021

\begin{abstract}
Summary
Background Correct recognition of risk factors enables individualized management and treatment of venom allergic patients.

Methods Systematic research and review of current literature regarding the risk of (1) severe sting-induced anaphylaxis, (2) anaphylactic adverse event during venom immunotherapy (VIT), and (3) treatment failure.

Results and discussion (1) Mastocytosis is the most important risk factor for severe sting-induced anaphylaxis. Hereditary $\alpha$-tryptasemia was recently identified as a genetic predictor of severe reactions. Older age is clearly associated with an increased risk; the respective impact of defined cardiovascular comorbidities has yet to be determined. Recent data do not support an aggravation of venom-induced anaphylaxis by intake of $\beta$-blockers or angiotensin-converting enzyme (ACE) inhibitors. A higher risk in men can be attributed to more intensive exposure to stinging insects. (2) Anaphylactic side effects of VIT are most common during the buildup phase, particularly in the course of (ultra-)rush protocols involving a high number of injections and high cumulative daily doses. They are significantly more frequent during honeybee compared to Vespula VIT. Data supporting a negative effect of mastocytosis on the tolerability of VIT are scarce. Older age and cardiovascular medication are not associated with a higher incidence of VITinduced anaphylaxis. (3) Relapsing anaphylactic reactions to both field and challenge stings are signifi-
\end{abstract}

J. Stoevesandt, MD $(\bowtie) \cdot A$. Trautmann

Department of Dermatology, Venereology, and Allergy and

the Allergy Center Mainfranken, University Hospital

Würzburg, Josef-Schneider-Straße 2, 97080 Würzburg,

Germany

Stoevesandt_J@ukw.de cantly more common during and after honeybee compared to Vespula VIT. Reports of severe field-sting reactions in mastocytosis patients suggest an increased risk of treatment failure which may be overcome by higher maintenance doses and longer duration of VIT.

Keywords ACE inhibitor · Age · Beta-blocker · Hereditary alpha-tryptasemia · Immunotherapy . Mastocytosis

\section{Abbreviations \\ VIT Venom immunotherapy}

\section{Introduction}

Hymenoptera stings belong to the most important elicitors of anaphylaxis worldwide. Most venom-induced anaphylactic reactions can be attributed to stings by honeybees or Vespula species in Northern European countries where the lifetime prevalence of sting-induced anaphylaxis has been reported to reach up to $7.5 \%$ in adults [1]. Approximately one third (17.5-42.0\%) [2-8] of sting-induced anaphylactic reactions are classified as severe; the broad range presumably reflects the retrospective approach of most studies assessing anaphylactic sting reactions and use of different severity grading systems [9]. Fatal outcome of sting-related anaphylaxis is overall rare with a reported annual rate of approximately 0.1 cases per million population in Europe, Australia, and North America [10]. A significant proportion of sting-induced fatalities, however, is likely to go undetected, especially if the causative sting is not witnessed by caregivers or bystanders, or mucocutaneous signs of anaphylaxis (urticaria, flush, or angioedema) are inconspicuous or absent. To complicate matters even further, up to $60 \%$ of sting-induced fatalities affect individuals who were presumably unaware of being 
allergic to insect venom [11]. The likelihood of a severe or fatal anaphylactic sting reaction is increased by certain risk factors which will be the primary focus of this article. The risk factors for anaphylactic side effects of venom immunotherapy (VIT), and treatment failure-defined as a relapsing sting reaction despite adequate treatment-overlap to some extent, but are not identical (Fig. 1). A separate section of this article reviewing the recent medical literature is thus dedicated to each of these situations. Clinical consequences and coping strategies to be considered in the presence of risk factors are summarized in Table 1.

\section{Methods}

A literature search was performed via PubMed using combinations of the terms age, anaphylaxis, angiotensin-converting enzyme inhibitor, antihypertensive, beta-blocker, cardiovascular, challenge sting, epinephrine, exercise, fatal, field sting, honeybee, Hymenoptera, immunotherapy, Kounis syndrome, mastocytosis, medication, Polistes, risk factor, severity, sting, tryptase, venom, vespid and Vespula. The search included articles in English and German language.
Articles from 2010-2021 were included preferentially, older data if considered of particular interest.

\section{Severe sting reactions}

The sting leading to the first-or in case of several episodes most severe-systemic reaction prior to the initiation of VIT is referred to as the "index sting." The severity of relapsing anaphylaxis due to future restings generally corresponds to that of the index sting reaction, meaning that patients who previously suffered a severe reaction will remain at increased risk $[12,13]$. In accordance with these findings, international treatment guidelines recommend VIT for patients with a history of moderate-to-severe sting-induced anaphylaxis [1, 14, 15], correct recognition of which is therefore essential.

Severe sting-induced anaphylaxis is typically dominated by difficult-to-treat cardiovascular symptoms [13], whereas mucocutaneous signs of anaphylaxis (urticaria, flush, or angioedema) are frequently inconspicuous or even absent. The missing report of skin symptoms during anaphylaxis - the reasons underlying this phenomenon are incompletely understood, but might include predominant activation

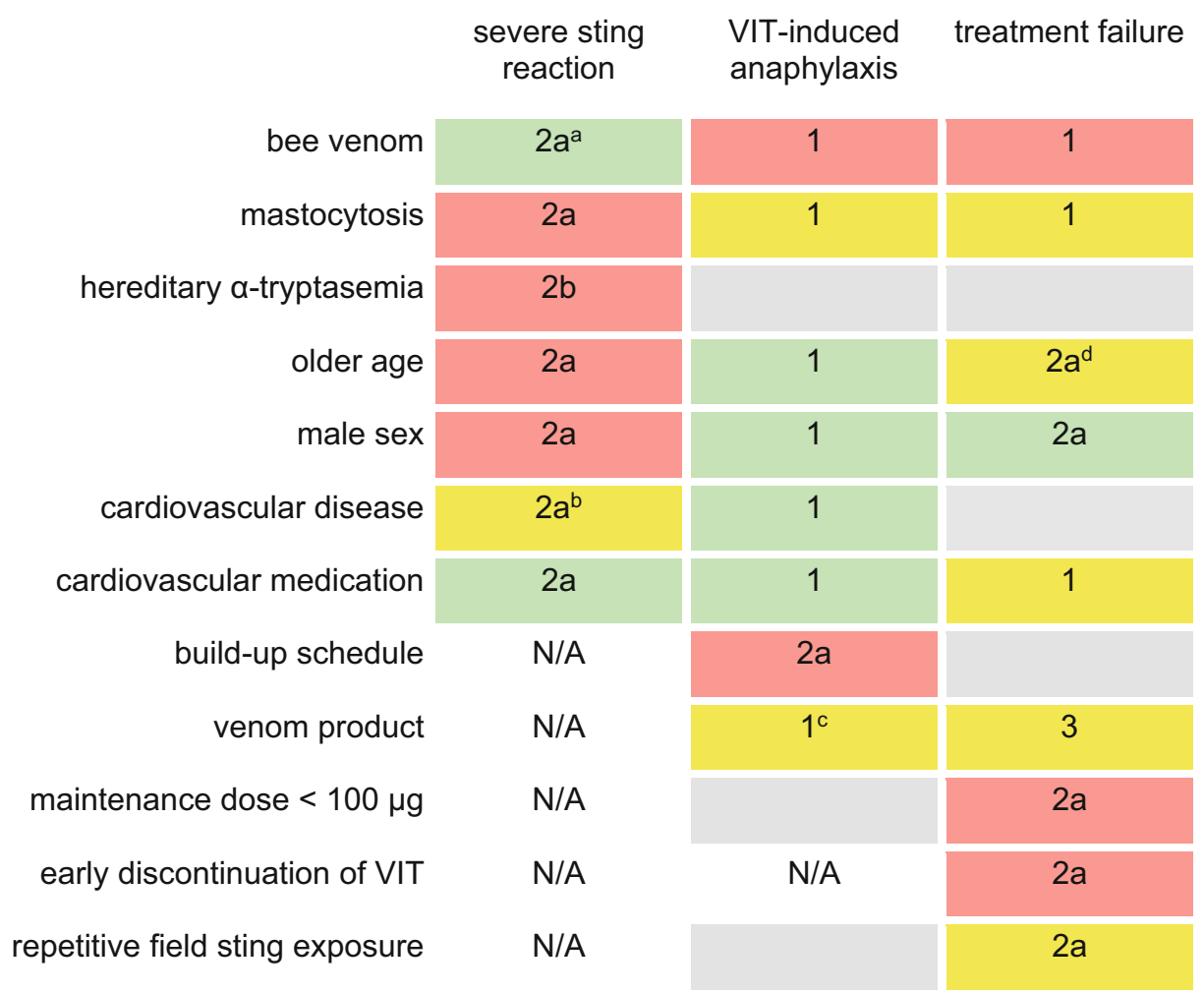

Fig. 1 Overview of risk factors in venom allergy. Risk level: red increased risk confirmed or likely, yellow controversial data, green increased risk excluded or unlikely, grey no data available, white (N/A) not applicable; level of evidence: 1 at least one prospective study, 2a several retrospective studies, $2 b$ one retrospective study, 3 small case series or case reports. ${ }^{a}$ Two studies suggest an increased risk of severe sting reactions in Vespula venom allergy [8, 24]. ${ }^{\mathrm{b}}$ Available data do not permit separate evaluation of defined cardiovascular conditions (e.g. coronary heart disease, cardiac insufficiency). 'Fewer large local reactions are observed when highly purified venom products are used; no definite statement can be made with regard to VIT-induced anaphylaxis [48]. dOIder age is not a risk factor per se, but relapse rates are lower in children and adolescents [12] 
Table 1 Risk factors in bee and Vespula venom allergy-clinical consequences and coping strategies

Risk of severe Be aware of high priority for VIT

sting-induced Prescribe epinephrine autoinjector ${ }^{\mathrm{a}}$

anaphylaxis? Minimize exposure

Provide advice on situative risk factors

Risk of VIT- Avoid build-up schedule involving high cumulative daily

induced ana- doses

phylaxis? Consider use of highly purified venom product

Consider antihistamine pretreatment

Consider temporary dose reduction ${ }^{b}$

Consider omalizumab treatment ${ }^{b}$

Risk of treat- Minimize exposure

ment failure? Arrange long-term prescription of epinephrine autoinjector

Consider increase of VIT maintenance dose

Consider prolongation of VIT

Re-consider choice of venom ${ }^{c}$

aprescription of two epinephrine autoinjectors is considered necessary for patients with previous near-fatal reaction or underlying mastocytosis [21]

${ }^{\mathrm{b}}$ Temporary dose reduction and/or off-label use of omalizumab [53] are not a preventive measure for risk reduction, but may be considered once VITinduced anaphylaxis has occurred.

'Use of venom product with higher content of icarapin might enhance VIT efficacy in bee venom allergic patients with dominant sensitization to Api $\mathrm{m} 10$ $[60,75]$

of cardiac mast cells and counter-regulatory release of endogenous epinephrine-is indicative not only of a severe reaction [2-5, 13], but also of basal serum tryptase elevation $[4,5,13]$ and underlying mastocytosis $[13,16,17]$. Rapid onset within minutes of the causative sting is another defining feature of severe venom-induced anaphylaxis [2-5, 13].

Risk factors for severe sting-induced anaphylaxis have been characterized in retrospective studies [2-8, $13,18]$ and information on fatal sting reactions is available from case series [11, 19, 20]. An obvious limiting factor of all mentioned studies is their inevitably retrospective approach (Fig. 1) as all anaphylactic sting reactions have occurred prior to data collection. Long-term risk factors for severe anaphylactic sting reactions are commonly unmodifiable and thus reinforce the indication for VIT and prescription of an emergency set-supply with two epinephrine autoinjectors is considered necessary for patients with previous near-fatal anaphylaxis and/or underlying mastocytosis (Table 1; [21]). Current knowledge on well-established, newly identified and controversial risk factors will be discussed in the following section.

\section{Situative risk factors/cofactors}

The term "cofactor" refers to short-term risk factors that lower the threshold and/or augment the severity of an anaphylactic reaction when coinciding with the eliciting trigger. Most of these parameters are-at least in theory-modifiable. An accurate knowledge of cofactors may thus enable allergic patients to actively avoid high risk situations. While there are ample data regarding the cofactors of food-, and particularly wheat-induced anaphylaxis [22, 23], information on situative risk factors in venom allergy is sparse. Regardless of the eliciting allergen, delayed administration of epinephrine has been associated with an unfavorable outcome of anaphylaxis [11]. Series of anaphylaxis fatalities including deaths from insect stings suggest that this also applies for delayed epinephrine treatment of anaphylactic sting reactions [20]. Of note, recent data from the European Anaphylaxis Registry demonstrate that adequate epinephrine treatment is less likely to be initiated in venom-induced anaphylaxis compared to anaphylactic reactions from other causes-especially in patients with hitherto undiagnosed venom allergy [13]. An aggravation of anaphylactic symptoms including cardiac deterioration and sudden death were also observed in patients who were forced to take a sitting or standing position in the course of an anaphylactic sting reaction [20]. Vigorous physical exercise is a well described cofactor in food allergy [23], and may likewise be relevant in all-cause anaphylaxis independent from the trigger factor [24]. Studies specifically assessing physical exercise as a cofactor in insect venom allergy are missing. Concurrent intake of acetyl salicylic acid, another established cofactor in wheat-induced anaphylaxis [22], was recently shown to be potentially relevant in venom-allergic reactions [13]. There are insufficient data regarding alcohol consumption as a cofactor in venom allergy.

\section{Mastocytosis}

There is abundant evidence confirming clonal mast cell disease as the leading risk factor for severe stinginduced anaphylaxis [18, 25-27], which typically goes along with severe protracted hypotension $[16,17]$ and may entail loss of consciousness and cardiac complications [13]. This risk is highest, though not confined to a distinct subgroup of predominantly male patients with indolent systemic mastocytosis characterized by an otherwise paucisymptomatic course, the absence of cutaneous mast cell infiltrates, and only mild to moderate basal serum tryptase elevation [28]. This is in accordance with the finding that tryptase values of less than $11.4 \mu \mathrm{g}$ - the 95th percentile arbitrarily used as a cut-off value-do not exclude underlying mastocytosis nor the risk of a severe systemic sting reaction [16]. Notwithstanding the above, basal serum tryptase elevation, as it reflects an increased individual mast cell burden or activity, is and remains an important indicator of a potentially severe anaphylactic sting reaction and is thus highly relevant in routine diagnostic risk assessment $[4-8,18]$.

\section{Hereditary $\alpha$-tryptasemia}

Hereditary $\alpha$-tryptasemia-a relatively common genetic trait with an estimated prevalence of $5.5 \%$ in the general population-is caused by an increased number of germline copies of $\alpha$-tryptase-encoding sequences at TPSAB1 and goes along with moder- 
ately elevated basal serum tryptase levels. Recent observations suggest an increased incidence of both systemic mastocytosis and severe systemic sting reactions-hereditary $\alpha$-tryptasemia might thus constitute the hitherto first genetic risk factor for severe stinginduced anaphylaxis [29]. The increased risk of severe anaphylaxis was attributed to naturally forming $\alpha-/ \beta$ tryptase heterotetramers [30], which were demonstrated to increase protease-activated receptor-2dependent endothelial permeability in vitro [29].

\section{Sex}

A predominance of male subjects ranging around $55 \%$ is consistently observed in cohorts of venomallergic patients [5-8], and an increased risk of severe reactions in men compared to women was detected by some [8] - though not all [5]—groups. This male overhang, which is also reflected in series of fatal sting reactions $[11,19,20]$, most likely results from more intensive exposure to stinging insects due to gender-specific occupational or leisure outdoor activities. In accordance with this assumption, a pronounced predominance of men $(84.4 \%)$ was found in a recent study assessing allergy and sensitization to Hymenoptera venoms in a cohort of fishers and hunters [31].

\section{Causative venom}

Some authors observed more severe systemic sting reactions in patients allergic to vespid venom compared to those allergic to bee venom $[8,24]$. Though this finding was not consistently confirmed by other groups $[4,5,18]$, there is no indication of the contrary, meaning that-despite its association with VITinduced anaphylactic reactions and higher rates of treatment failure (see below)-honeybee venom allergy is not a risk factor for severe sting-induced anaphylaxis.

\section{Older age}

Older age is associated with an increased risk of both severe anaphylactic reactions in general and severe sting reactions in particular $[4-8,13,18]$. Accordingly, severe sting-induced anaphylaxis is considerably less common in children and adolescents [32, 33]. While the mechanism underlying this age-dependent increase of severe anaphylactic sting reactions is incompletely understood, it appears reasonable to assume that children and young adults are generally more healthy, whereas comorbidity and particularly cardiovascular disease become more frequent with increasing age [33]. The tight connection between older age, cardiovascular comorbidity and cardiovascular medication, however, impairs separate statistical evaluation of these parameters [34]. Several authors have observed an age-dependent rise of basal serum tryptase concentrations as a potential indicator of an increasing mast cells burden or mast cell activity [6, 7, 33].

\section{Cardiovascular disease}

Data on all-cause anaphylactic reactions - that is independent of the eliciting allergen-suggest a more severe course in patients with cardiac comorbidity as compared to healthy controls [35, 36], possibly due to an impaired compensation of mast-cell mediated cardiac symptoms [37]. Moreover, severe anaphylaxis may coincide with cardiac ischemia or even myocardial infarction. Acute coronary syndrome as a complication of anaphylaxis is referred to as "Kounis syndrome" and has been described in venom allergy by several authors [38, 39]. In accordance with these observations, autopsy findings revealed cardiovascular shock and circulatory arrest as the most frequent cause of death in fatal sting reactions [40]. Somewhat surprisingly, several retrospective studies specifically assessing risk factors for severe systemic sting reactions did not confirm an aggravation of venom-induced anaphylaxis by cardiovascular comorbidity [5, 8, 18], whereas recent data originating from the European Anaphylaxis Registry suggest an unfavorable impact [13]. An obvious limiting factor of all mentioned studies is that cardiovascular comorbidity was assessed as a whole, meaning that no differentiation was made between mere arterial hypertension and potentially more relevant cardiac conditions such as coronary artery disease or chronic heart failure. Sufficiently large studies considering distinct cardiac diseases will be required to clarify their respective impact on the severity of anaphylactic sting reactions. In the meantime, venom allergic cardiac patients should be considered at an increased risk of a severe systemic sting reaction and thus as preferential candidates for VIT.

\section{Cardiovascular medication}

Other than the risk factors discussed above, antihypertensive medication is modifiable - it can be changed or discontinued and is thus a potential target for risk reduction. Safety concerns regarding the use of antihypertensive drugs in venom-allergic patients were brought up in the 1980s based on case reports suggesting an aggravation of sting-induced systemic reactions by beta-blockers [41], supposedly by counteracting the effects of endogenously released and therapeutically administered catecholamines. Recent retrospective studies specifically assessing risk factors for severe systemic sting reactions, however, did not confirm an unfavorable effect of beta-blockers [5, 8, 18]. The focus of interest subsequently moved towards angiotensin-converting enzyme (ACE) inhibitors, which were observed to potentially aggravate sting-induced anaphylaxis by several $[8,13]$, but not all $[4,5]$ groups. 
ACE inhibitor-mediated augmentation of anaphylaxis was theoretically explained by impaired degradation of anaphylaxis mediator bradykinin and/or a direct interaction of ACE inhibitor and mast cell, and an additive negative effect of both ACE inhibitors and betablockers was observed in a mouse model [42]. Retrospective studies assessing all-cause anaphylaxis were likewise suggestive of an aggravation by cardiovascular medication [13, 24, 42]. Effects of multicollinearity arising from the close association of antihypertensive medication with both older age and cardiac comorbidity, however, impair statistical evaluation of cardiovascular medication as an individual predictor of severe anaphylaxis. Accordingly, an overall low quality of evidence due to insufficient control of confounders was ascertained in a recent meta-analysis investigating observational studies on the relationship between anaphylaxis and cardiovascular medication [34]. This problem was recently addressed in an observational multicenter trial including a sufficiently large cohort of patients ( $n=1425$ ) - study data provide robust evidence that taking beta-blockers or ACE inhibitors is not an independent risk factor for severe systemic sting reactions [18]. Leaving aside these considerations, any recommendation to change or discontinue an established antihypertensive medication in order to minimize the risk of a severe sting reaction needs to be weighed against the benefit of guideline-directed treatment on the morbidity and mortality of the underlying cardiovascular disease [34, 43].

\section{Anaphylactic side effects of VIT}

VIT goes along with repetitive exposure to the causative venom and is thus a potential elicitor not only of large local reactions, but also occasionally of anaphylaxis [44]. Available data on anaphylactic side effects of VIT are based on a number of retrospective [33, 43, 45, 46] and prospective [18, 47-49] studies (Fig. 1) of varying size and design. The overall incidence of VIT-induced systemic reactions reaches $3-20 \%$ [18, $43,45-47,49]$. The broad range is presumably due to the use of different anaphylaxis grading systems [50, 51] and inconsistent definition of anaphylactic side effects, including subjective reactions in some studies. Additional factors (e.g. choice of therapeutic venom preparation and treatment protocol) that influence the incidence of treatment-related side effects and may vary between study centers will be discussed below.

The majority of objective VIT-related systemic reactions present as isolated urticaria and/or angioedema. Mucocutaneous symptoms may develop with a delay of several hours following VIT injections and usually respond well to antiallergic treatment [50]. Premedication with antihistamines may be considered in order to prevent this inconvenient but controllable side effect of VIT [52]. Full-blown anaphylaxis involving one or several extracutaneous organs is less com- mon [18, 43, 45, 46, 49, 50]. It usually evolves within minutes of VIT injections and may take a dynamic course [50]. Still, adequate monitoring and antiallergic treatment generally enable effective control, and the $100 \mu \mathrm{g}$ standard maintenance dose can be safely reached and maintained in the majority of affected patients $[43,50]$. Off-label medication with anti-IgE antibodies may be considered in the rare case of repetitive VIT-induced anaphylaxis in order to enable continuation of treatment [53]. Fatal VIT-related anaphylaxis is a rarity and has only been observed anecdotally. Published case reports documented a questionable relation between VIT and fatal reaction due to either co-administration of other allergens [54], or discontinuation of essential cardiovascular drugs as an alternative trigger of fatal ventricular arrhythmia [55]. The history of a severe index-sting reaction is not predictive of VIT-related side effects [18, 43, 47].

\section{Causative venom}

Studies assessing the safety of VIT consistently found that anaphylactic complications are more frequent during treatment with honeybee compared to Vespula venom [18, 33, 43-47, 49]. Inferior tolerability of bee venom presumably results from its more complex composition and higher number of relevant allergens, the respective content of which significantly differs between venom products from different manufacturers [56]. One group observed a higher rate of bee VIT-induced anaphylactic reactions in patients with predominant sensitization to melittin (Api m 4) [57].

\section{Therapeutic venom preparation}

There is a lack of studies comparing the safety profile of commercial venom preparations provided by different manufacturers. All therapeutic venom products are derived from natural honeybee or Vespula venom, but information specifying the respective steps of processing and purification is scarce [56]. Available data suggest a better tolerability and especially a lower rate of large local reactions at the injection site if highly purified venom products - this does include ALK-lyophilisiert SQ ${ }^{\mathrm{TM}}$ (ALK-Abelló Arzneimittel $\mathrm{GmbH}$, Hamburg, Germany, referred to as Aquagen ${ }^{\mathrm{TM}}$ in some countries)-are used [48]. This has been attributed to a reduced content of low-molecular components and bioactive peptides [48, 58]. Conversely, venom preparations undergoing less intensive purification-as for example ALK Reless ${ }^{\mathrm{TM}}$ (ALK-Abelló Arzneimittel GmbH, Hamburg, Germany, internationally referred to as Pharmalgen ${ }^{\mathrm{TM}}$ ), Bencard Venomil ${ }^{\mathrm{TM}}$ (Bencard Allergie $\mathrm{GmbH}$, München, Germany), and HAL Allergy Venomenhal ${ }^{\mathrm{TM}}$ (HAL Allergie GmbH, Düsseldorf, Germany) may contain a broader and more representative spectrum of different venom allergens, which might represent a therapeutic advantage, especially in the case of honeybee venom (see below 
for details) [56, 59, 60]. Superior tolerability was also documented for aluminum hydroxide adsorbed depot preparations such as ALK depot SQ ${ }^{\mathrm{TM}} /$ Alutard $\mathrm{SQ}^{\mathrm{TM}}$ (ALK-Abelló Arzneimittel GmbH, Hamburg, Germany, internationally referred to as Alutard $\mathrm{SQ}^{\mathrm{TM}}$ ) which are available for conventional dose increase and maintenance of VIT [58].

\section{Treatment protocol}

Anaphylactic reactions most frequently occur during the build-up phase of VIT [49] once doses exceeding $20 \mu \mathrm{g}$ have been reached [61]. They are more common in the course of rush or ultra-rush schedules compared to conventional build-up [47, 49], especially in protocols involving a critical number of single injections [46] and high cumulative daily doses [43]. A higher incidence of VIT-induced systemic reactions was likewise observed during VIT build-up according to cluster protocols by some authors [62].

\section{Mastocytosis}

It is generally assumed that concurrent mastocytosis goes along with an increased incidence and possibly an augmented severity of VIT-induced anaphylaxis [27]. Studies evaluating the safety of VIT in venom allergic mastocytosis patients, however, yielded controversial results. An increased incidence of VIT-related adverse reactions in the case of underlying mastocytosis was observed in a number of relatively small retrospective case series [63, 64]. Accordingly, Ruëff et al. described a log-linear association between increasing baseline serum tryptase levels and the necessity of anti-allergic treatment in the course of VIT build-up with vespid venom [47]. Other works including the recent prospective multicenter study by Sturm et al. did not confirm an effect of basal serum tryptase concentration or underlying mastocytosis [18]. Of note, none of the mentioned studies focusing on the overall incidence of VIT-induced adverse events permits any conclusion regarding their respective severity, meaning that no definite statement can be made on whether or not VIT-related anaphylaxis may be aggravated by underlying mastocytosis. Still, there is broad consensus that the benefit of VIT outweighs its risks, also and especially in mastocytosis patients who are at substantial risk of severe and potentially fatal anaphylactic sting reactions (see above), whereas VIT-induced anaphylaxis is both calculable and treatable [27, 63-65].

\section{Age and sex}

In some contrast to the finding of an age-dependent aggravation of field-sting induced anaphylaxis (see above), there is no evidence of a higher incidence or greater severity of VIT-related anaphylactic adverse events in older age [18, 43, 49]. On the contrary, the frequency of VIT-induced anaphylaxis was found to gradually decrease with age by some groups [47], and others observed an increased incidence in children and adolescents compared to adult controls [33]. Minor age, however, was not confirmed as an independent risk factor as the higher frequency of treatment-induced systemic reactions in children was attributed to a greater proportion of bee-venom allergic patients and thus to the inferior tolerability of bee venom products [33]. Women were suggested to be at an increased risk of VIT-related anaphylaxis by some groups [49], while a significant sex-related effect was not observed by others [43, 47].

\section{Cardiovascular medication and cardiovascular disease}

An aggravating effect of cardiovascular medication, and particularly of ACE inhibitors and beta-blockers on the incidence or the severity of VIT-related anaphylactic reactions has long been the subject of controversial discussion, but is to date only supported on the level of case reports [66]. Studies on risk factors for VIT-induced anaphylaxis-including but not restricted to the recent prospective European multicenter study by Sturm et al. [18, 43, 45, 47, 55]—consistently did not confirm an association between cardiovascular medication and the incidence of VIT-related anaphylaxis. Available data thus do not justify modification of an established antihypertensive treatment prior to the initiation of VIT for safety reasons. Due to the lack of respective data, no definite statement, however, can to date be made regarding a potential influence of cardiovascular medication on the severity of VIT-induced anaphylaxis. The same applies for concurrent arterial hypertension or cardiac comorbidity which were not found to go along with an increased incidence of VIT-induced anaphylactic side effects $[18,50]$.

\section{Treatment failure}

Allergen-specific tolerance resulting from subcutaneous VIT enables effective and longstanding protection from sting-induced anaphylaxis for the majority of patients [44]. The overall incidence of treatment failure is about 5\% during ongoing VIT [44, 67]. Whereas field sting reactions during or after VIT are generally expected to be of lesser severity than the initial index sting reaction, they may occasionally take a severe or even fatal course [68, 69]. Sting challenges have been advocated as the diagnostic gold standard to monitor the effectiveness of VIT and are widely practiced in German-speaking countries [1, 70-72], albeit less on an international level [14, 15]. Reproducibility of a single field sting and thus its predictive value for the individual patient remain a debated issue [1]. Large series of sting challenges during the maintenance phase of VIT, however, provide a sound statistical basis for the identification 
of risk factors for treatment failure and are thus of scientific importance [67]. Both the actual incident of an anaphylactic relapse reaction due to a field or challenge sting, and the presence of risk factors predictive of treatment failure, require consideration of measures for risk reduction (Table 1) including dose increase, prolongation of VIT and long-term supply with an emergency set [21]. The risk of treatment failure is higher in patients developing an anaphylactic adverse event during VIT $[67,73]$, further risk factors will be discussed in detail below.

\section{Causative venom and venom preparation}

Honeybee VIT is clearly associated with a higher risk of relapsing sting reactions as compared to Vespula VIT $[67,73,74]$. This might in part be due to the larger amount of venom toxin delivered by a bee sting [1]. Moreover, honeybee venom contains a larger number of potentially relevant allergens, and sensitization profiles differ between individual patients. In particular, dominant sensitization to Api m 10 (icarapin), an instable bee venom allergen of low abundance, has been identified as a potential predictor of treatment failure [59]. Due to product-specific methods of processing and purification, commercial venom preparations considerably differ with regard to their respective content of Api m 10 [59], and possibly also of Api m 3 and Api m 5 [56], which may be underrepresented in the final therapeutic product. Hypothetically, treatment with a venom product of "lesser" purification and thus higher content of instable Api m 10 may optimize protection in bee venom allergic patients with dominant sensitization to icarapin [60]. Evidence supporting this approach, however, is to date restricted to the level of case reports $[60,75]$.

\section{Dose and duration of VIT}

VIT efficacy is dose-dependent, and an increased rate of treatment failure is to be expected if only $50 \mu \mathrm{g}$ per injection are given during VIT maintenance instead of the $100 \mu \mathrm{g}$ standard dose [76]. Accordingly, increasing the maintenance dose from 100 to $200 \mu \mathrm{g}$ is considered to further optimize protection $[67,70]$. Dose increase is recommended in case of a relapsing sting reaction while on standard $100 \mu \mathrm{g}$ maintenance VIT $[1,70,71]$ and may also be adequate for venom allergic bee keepers at risk of multiple stings and for mastocytosis patients $[1,70]$.

Protection is established shortly after updosing of VIT [77], is considered to be optimal several months into the maintenance phase [67] and to partially decrease following VIT discontinuation [74]. Premature discontinuation of VIT after only 1 or 2 years has been associated with an increased risk of relapse [78], whereas 3 to 5 years of treatment are considered sufficient for most patients [74, 79]. Extended prolongation of VIT is generally recommended for mastocytosis patients [27] and in case of high exposure due to ongoing bee keeping activities. Based on an individual risk assessment, it may also be appropriate in patients with very severe index sting reactions or systemic adverse events during VIT [1].

\section{Repetitive exposure}

The assumption that patients undergoing multiple restings are at an increased risk of treatment failure $[74,80]$ is plausible, but not without controversy as it could not be reproduced in series of repetitive challenge stings following VIT discontinuation [79].

\section{Mastocytosis}

Clonal mast cell disease is presumably associated with a higher incidence of anaphylactic field stings reactions during VIT [64] or after its discontinuation [27, 68]. This is in line with the observation that a severe initial index sting reaction might be predictive of future treatment failure [78]. Evidence supporting this assumption, however, is relatively poor. Ruëff et al. did not observe an association between baseline serum tryptase elevation and the rate of treatment failure in 357 patients undergoing sting challenges $(n=154)$ or reporting field stings $(n=203)$ during the maintenance phase of VIT [73], but suggested tryptase values exceeding $20 \mu \mathrm{g} / \mathrm{L}$ and concurrent cutaneous signs of systemic mastocytosis to be predictive of systemic reactions to challenge stings in a subsequent publication [67].

Irrespective of its overall incidence, relapsing stinginduced anaphylaxis may take a severe (and occasionally fatal) course in mastocytosis patients $[27,63,68$, 69]. As a consequence, most [1, 14] though not all [15] international guidelines on VIT recommend prolonged or even life-long treatment in case of concurrent mastocytosis.

\section{Age and sex}

There no indication of sex being a predictor for treatment failure $[67,73]$. Older age was not identified as a risk factor per se [67, 73], but long-term outcome in children is considered to be superior compared to adults [12].

\section{Cardiovascular medication and cardiovascular disease}

An increased risk of relapsing sting reactions has been discussed for patients receiving antihypertensive treatment, especially with ACE inhibitors or betablockers. Available data, however, are inconsistent. Whereas no cases of relapsing anaphylaxis were found in patients taking beta-blockers $(n=9)$ or ACE inhibitors $(n=15)$ upon re-exposure to stings of the culprit insect in the 2013 prospective observational 
multicenter study by Ruëff et al. [73], an association of ACE inhibitor medication and treatment failure was suggested in a retrospective study examining a considerably larger, but overlapping series of patients undergoing a sting challenge [67] - the latter might be biased by preselection of high-risk patients as both ACE inhibitors and beta-blockers were discontinued prior to the sting challenge whenever considered justifiable by a cardiologist. No indication of an increased incidence or severity of relapsing sting-induced anaphylaxis in patients taking ACE inhibitors or betablockers was found in the prospective multicenter study by Sturm et al. upon evaluation of 210 re-stings during VIT maintenance [18]. Due to the lack of respective data, no statement can be made with regard to cardiovascular disease as a risk factor for treatment failure.

\section{Conclusions}

- Risk factors for severe systemic sting reactions, anaphylactic side effects of VIT (venom immunotherapy), and treatment failure overlap, but are not identical.

- Indolent systemic mastocytosis and older age are the main risk factors for severe sting-induced anaphylaxis; thus, they reinforce the indication for VIT.

- Cardiovascular medication does not increase the incidence of VIT-induced anaphylactic reactions.

- The potential effect of defined cardiovascular conditions on the severity and outcome of anaphylactic sting reactions has yet to be assessed.

- Honeybee venom allergy is the most relevant risk factor for both anaphylactic reactions during VIT and treatment failure.

- Full-blown anaphylaxis is a rare and generally controllable complication of VIT.

- Treatment failure may be overcome by use of higher maintenance doses and prolongation of VIT.

Funding Open Access funding enabled and organized by Projekt DEAL.

Conflict of interest J. Stoevesandt declares that she has no competing interests. A. Trautmann reports consultancy fees from ALK-Abelló, outside of the submitted work.

Open Access This article is licensed under a Creative Commons Attribution 4.0 International License, which permits use, sharing, adaptation, distribution and reproduction in any medium or format, as long as you give appropriate credit to the original author(s) and the source, provide a link to the Creative Commons licence, and indicate if changes were made. The images or other third party material in this article are included in the article's Creative Commons licence, unless indicated otherwise in a credit line to the material. If material is not included in the article's Creative Commons licence and your intended use is not permitted by statutory regulation or exceeds the permitted use, you will need to obtain permission directly from the copyright holder. To view a copy of this licence, visit http://creativecommons.org/licenses/by/4.0/.

\section{References}

1. Sturm GJ, Varga EM, Roberts G, Mosbech H, Bilò MB, Akdis CA, et al. EAACI guidelines on allergen immunotherapy: hymenoptera venom allergy. Allergy. 2018;73:744-64.

2. Chapsa M, Roensch H, Langner M, Beissert S, Bauer A. Predictors of severe anaphylaxis in hymenoptera venom allergy: the importance of absence of urticaria and angioedema. Ann Allergy Asthma Immunol. 2020;125:72-7.

3. Fehr D, Micaletto S, Moehr T, Schmid-Grendelmeier P. Risk factors for severe systemic sting reactions in wasp (Vespula spp.) and honeybee (Apis mellifera) venom allergic patients. Clin Transl Allergy. 2019;9:54.

4. Arzt L, Bokanovic D, Schwarz I, Schrautzer C, Massone C, Horn $\mathrm{M}$, et al. Hymenoptera stings in the head region induce impressive, but not severe sting reactions. Allergy. 2016;71:1632-4.

5. StoevesandtJ, Hain J, KerstanA, TrautmannA. Over-and underestimated parameters in severe hymenoptera venominduced anaphylaxis: cardiovascular medication and absence of urticaria/angioedema. J Allergy Clin Immunol. 2012;130:698-704.

6. Blum S, Gunzinger A, Müller UR, Helbling A. Influence of total and specific IgE, serum tryptase, and age on severity of allergic reactions to hymenoptera stings. Allergy. 2010;66:222-8.

7. Guenova E, Volz T, Eichner M, Hoetzenecker W, Caroli U, Griesinger $\mathrm{G}$, et al. Basal serum tryptase as risk assessment for severe hymenoptera sting reactions in elderly. Allergy. 2010;65:919-23.

8. Ruëff F, Przybilla B, Bilò MB, Müller U, Scheipl F, Aberer W, etal. Predictors of severe systemic anaphylactic reactions in patients with hymenoptera venom allergy: importance of baseline serum tryptase-a study of the European Academy of Allergology and Clinical Immunology Interest Group on Insect Venom Hypersensitivity. J Allergy Clin Immunol. 2009;124:1047-54.

9. Muraro A, Fernández-Rivas M, Beyer K, Cardona V, ClarkA, Eller E, et al. The urgent need for a harmonized severity scoring system for acute allergic reactions. Allergy. 2018;73:1792-800.

10. Turner PJ, Jerschow E, Umasunthar T, Lin R, Campbell DE, Boyle RJ. Fatal anaphylaxis: mortality rate and risk factors. JAllergy Clin Immunol Pract. 2017;5:1169-78.

11. Xu YS, Kastner M, Harada L, Xu A, Salter J, Waserman S. Anaphylaxis-related deaths in Ontario: a retrospective review of cases from 1986 to 2011. Allergy Asthma Clin Immunol. 2014;10:38.

12. Golden DB, Kagey-Sobotka A, Norman PS, Hamilton RG, Lichtenstein LM. Outcomes of allergy to insect stings in children, with and without venom immunotherapy. NEngl JMed. 2004;351:668-74.

13. Francuzik W, Ruëff F, Bauer A, Bilò MB, Cardona V, Christoff G, et al. Phenotype and risk factors of venominduced anaphylaxis: a case-control study of the European Anaphylaxis Registry. J Allergy Clin Immunol. 2021;147(2):653-662.e9.

14. Golden DB, Demain J, Freeman T, Graft D, Tankersley M, Tracy J, et al. Stinging insect hypersensitivity: a practice parameter update 2016. Ann Allergy Asthma Immunol. 2017;118:28-54.

15. Krishna MT, Ewan PW, Diwakar L, Durham SR, Frew AJ, Leech SC, et al. Diagnosis and management of hymenoptera venom allergy: British Society for Allergy and Clinical Immunology (BSACI) guidelines. Clin Exp Allergy. 2011;41:1201-20. 
16. Zanotti R, Lombardo C, Passalacqua G, Caimmi C, Bonifacio M, De Matteis G, et al. Clonal mast cell disorders in patients with severe hymenoptera venom allergy and normal serum tryptase levels. J Allergy Clin Immunol. 2015;136:135-9.

17. Álvarez-Twose I, González de Olano D, Sánchez-Muñoz L, Matito A, Jara-Acevedo M, Teodósio C, etal. Validation of the REMA score for predicting mast cell clonality and systemic mastocytosis in patients with systemic mast cell activation symptoms. Int Arch Allergy Immunol. 2012;157:275-80.

18. Sturm GJ, Herzog SA, Aberer W, Alfaya Arias T, AntolínAmérigo D, Bonadonna P, et al. Beta-blockers and ACE inhibitors are not a risk factor for severe systemic sting reactions and adverse events during venom immunotherapy. Allergy. 2021;76:2166-76.

19. Jerschow E, Lin RY, Scaperotti MM, McGinn AP. Fatal anaphylaxis in the United States, 1999-2010: temporal patterns and demographic associations. J Allergy Clin Immunol. 2014;134:1318-28.

20. Mullins RJ, Wainstein BK, Barnes EH, Liew WK, Campbell $D E$. Increases in anaphylaxis fatalities in Australia from 1997 to 2013. Clin Exp Allergy. 2016;46:1099-110.

21. Bilò MB, Cichocka-Jarosz E, Pumphrey R, Oude-ElberinkJN, Lange J, Jakob T, et al. Self-medication of anaphylactic reactions due to hymenoptera stings-an EAACI task force consensus statement. Allergy. 2016;71:931-43.

22. Christensen MJ, Eller E, Mortz CG, Brockow K, BindslevJensen $\mathrm{C}$. Wheat-dependent cofactor-augmented anaphylaxis: a prospective study of exercise, aspirin, and alcohol efficacy as cofactors. J Allergy Clin Immunol Pract. 2019;7:114-21.

23. Wölbing F, Fischer J, Köberle M, Kaesler S, Biedermann T. About the role and underlying mechanisms of cofactors in anaphylaxis. Allergy. 2013;68:1085-92.

24. Worm M, Francuzik W, Renaudin JM, Bilò MB, Cardona V, Scherer Hofmeier K, et al. Factors increasing the risk for a severe reaction in anaphylaxis: an analysis of data from the European Anaphylaxis Registry. Allergy. 2018;73:1322-30.

25. Vos B, van Anrooij B, van Doormaal JJ, Dubois AEJ, Oude Elberink JNG. Fatal anaphylaxis to yellow jacket stings in mastocytosis: optionsforidentification and treatment ofatrisk patients. JAllergy Clin Immunol Pract. 2017;5:1264-71.

26. Gülen T, Hägglund H, Dahlén B, Nilsson G. High prevalence of anaphylaxis in patients with systemic mastocytosis-a single-centre experience. Clin Exp Allergy. 2014;44:121-9.

27. Niedoszytko M, de Monchy J, van Doormaal JJ, Jassem E, Oude Elberink JN. Mastocytosis and insect venom allergy: diagnosis, safety and efficacy of venom immunotherapy. Allergy. 2009;64:1237-45.

28. Álvarez-Twose I, Zanotti R, González-de-Olano D, Bonadonna P, Vega A, Matito A, et al. Nonaggressive systemic mastocytosis (SM) without skin lesions associated with insect-induced anaphylaxis shows unique features versus other indolent SM. J Allergy Clin Immunol. 2014;133:520-8.

29. Lyons JJ, Chovanec J, O'Connell MP, Liu Y, Selb J, Zanotti R, et al. Heritable risk for severe anaphylaxis associated with increased alpha-tryptase-encoding germline copy number at TPSAB1. JAllergy Clin Immunol. 2021;147:622-32.

30. Le QT, Lyons JJ, Naranjo AN, Olivera A, Lazarus RA, Metcalfe DD, et al. Impact of naturally forming human alpha/beta-tryptase heterotetramers in the pathogenesis of hereditary alpha-tryptasemia. J Exp Med. 2019;216:2348-61.

31. Zink A, Schuster B, Winkler J, Eyerich K, Darsow U, Brockow K, et al. Allergy and sensitization to hymenoptera venoms in unreferred adults with a high risk of sting exposure. World Allergy Organ J. 2019;12:100039.

32. Golden DB. Insectallergy in children. Curr Opin Allergy Clin Immunol. 2006;6:289-93.

33. Stoevesandt J, Hosp C, Kerstan A, Trautmann A. Safety of $100 \mu \mathrm{g}$ venom immunotherapy rush protocols in children compared to adults. Allergy Asthma Clin Immunol. 2017;13:32.

34. Tejedor-Alonso MA, Farias-Aquino E, Perez-Fernandez E, Grifol-Clar E, Moro-Moro M, Rosado-Ingelmo A. Relationship between anaphylaxis and use of beta-blockers and angiotensin-converting enzyme inhibitors: a systematic review and meta-analysis of observational studies. JAllergy Clin Immunol Pract. 2019;7:879-897.e5.

35. Motosue MS, Bellolio MF, Van Houten HK, Shah ND, Campbell RL. Risk factors for severe anaphylaxis in the United States. Ann Allergy Asthma Immunol. 2017;119:356-61.

36. Müller UR. Cardiovascular disease and anaphylaxis. Curr Opin Allergy Clin Immunol. 2007;7:337-41.

37. Marone G, Genovese A, Varricchi G, Granata F. Human heart as a shockorgan in anaphylaxis. Allergo J Int. 2014;23:60-6.

38. Cross B, Choudhury TR, Hindle M, Galasko G. Wasp sting induced STEMI with complete coronary artery occlusion: a case of Kounis syndrome. BMJ Case Rep. 2017; https:// doi. org/10.1136/bcr-2017-221256.

39. Aminiahidashti H, Laali A, Samakoosh AK, Gorji AM. Myocardial infarction following a bee sting: a case report of Kounis syndrome. Ann Card Anaesth. 2016;19:375-8.

40. Pumphrey RS. Lessons for management of anaphylaxis from a study of fatal reactions. Clin Exp Allergy. 2000;30:1144-50.

41. Ingall M, Goldman G, Page LB. Beta-blockade in stinging insect anaphylaxis. JAMA. 1984;251:1432.

42. Nassiri M, Babina M,DölleS, EdenharterG, RuëffF, Worm M. Ramipril and metoprolol intake aggravate human and murine anaphylaxis: evidence for direct mast cell priming. JAllergy Clin Immunol. 2015;135:491-9.

43. Stoevesandt J, Hain J, Stolze I, Kerstan A, Trautmann A. Angiotensin-converting enzyme inhibitors do not impair the safety of hymenoptera venom immunotherapy buildup phase. Clin Exp Allergy. 2014;44:747-55.

44. Boyle RJ, Elremeli M, Hockenhull J, Cherry MG, Bulsara MK, Daniels $\mathrm{M}$, et al. Venom immunotherapy for preventing allergic reactions to insect stings. Cochrane Database Syst Rev. 2012;10:CD8838.

45. Stoevesandt J, Hosp C, Kerstan A, Trautmann A. Hymenoptera venom immunotherapy while maintaining cardiovascular medication: safe and effective. Ann Allergy Asthma Immunol. 2015;114:411-6.

46. Brehler R, Wolf H, Kütting B, Schnitker J, Luger T. Safety of atwo-dayultrarushinsectvenomimmunotherapyprotocol in comparison with protocols oflonger duration and involving a larger number of injections. J Allergy Clin Immunol. 2000;105:1231-5.

47. Ruëff F, Przybilla B, Bilò MB, Müller U, Scheipl F, Aberer W, et al. Predictors of side effects during the buildup phase of venom immunotherapy for hymenoptera venom allergy: the importance of baseline serum tryptase. J Allergy Clin Immunol. 2010;126:105-11.

48. Bilò MB, Severino M, Cilia M, Pio A, Casino G, Ferrarini E, et al. The VISYT trial: venom immunotherapy safety and tolerability with purified vs nonpurified extracts. Ann Allergy Asthma Immunol. 2009;103:57-61.

49. Mosbech H, Müller U. Side-effects of insect venom immunotherapy: results from an EAACI multicenter study. European Academy of Allergology and Clinical Immunology. Allergy. 2000;55:1005-10. 
50. Stoevesandt J, Hosp C, Kerstan A, Trautmann A. Risk stratification of systemic allergic reactions during hymenoptera venom immunotherapy buildup phase. J Dtsch Dermatol Ges. 2014;12:244-55.

51. Cox L, Larenas-Linnemann D, Lockey RF, Passalacqua G. Speaking the same language: the World Allergy Organization subcutaneous immunotherapy systemic reaction grading system. J Allergy Clin Immunol. 2010;125:569-574.e7.

52. Reimers A, Hari Y, Müller U. Reduction of side-effects from ultrarush immunotherapy with honeybee venom by pretreatment with fexofenadine: a double-blind, placebocontrolled trial. Allergy. 2000;55:484-8.

53. Stretz E, Oppel EM, Rawer HC, Chatelain R, Mastnik S, Przybilla B, et al. Overcoming severe adverse reactions to venom immunotherapy using anti-IgE antibodies in combination with a high maintenance dose. Clin Exp Allergy. 2017;47:1631-9.

54. Lockey RF, Benedict LM, Turkeltaub PC, Bukantz SC. Fatalities fromimmunotherapy (IT) and skin testing (ST).JAllergy Clin Immunol. 1987;79:660-77.

55. Müller UR, Haeberli G. Use of beta-blockers during immunotherapy for hymenoptera venom allergy. JAllergy Clin Immunol. 2005;115:606-10.

56. Blank S, Etzold S, Darsow U, Schiener M, Eberlein B, Russkamp D, et al. Component-resolved evaluation of the content of major allergens in therapeutic extracts for specific immunotherapy of honeybee venom allergy. Hum Vaccin Immunother. 2017;13:2482-9.

57. Ruiz B, Serrano P, Verdu M, Moreno C. Sensitization to Api $\mathrm{m} 1$, Api $\mathrm{m} 2$, and Api $\mathrm{m}$ 4: association with safety of bee venom immunotherapy. Ann Allergy Asthma Immunol. 2015;114:350-2.

58. Bilò MB, Antonicelli L, Bonifazi F. Purified vs. nonpurified venom immunotherapy. Curr Opin Allergy Clin Immunol. 2010;10:330-6.

59. FrickM, Fischer J, HelblingA, RuëffF, WieczorekD, Ollert M, et al. Predominant Api m 10 sensitization as risk factor for treatment failure in honey bee venom immunotherapy. JAllergy Clin Immunol. 2016;138:1663-1671.e9.

60. Jakob T, Rauber MM, Perez-Riverol A, Spillner E, Blank S. The honeybee venom major allergen Api m 10 (Icarapin) and its role in diagnostics and treatment of hymenoptera venomallergy. Curr Allergy Asthma Rep. 2020;20:48.

61. Čerpes U, Arzt-Gradwohl L, Schrautzer C, Koch L, Bokanovic D, Laipold K, et al. Simultaneous up-dosing of bee and vespid venom immunotherapy is safe. Allergy. 2020;75:721-3.

62. Pospischil IM, Kagerer M, Cozzio A, Angelova-Fischer I, GuenovaE, Ballmer-Weber B, etal. Comparison of the safety profiles of 3 different hymenoptera venomimmunotherapy protocols: a retrospective 2-center study of 143 patients. Int Arch Allergy Immunol. 2020;181:783-9.

63. González de Olano D, Álvarez-Twose I, Esteban-López MI, Sánchez-Muñoz L, de Durana MD, Vega A, et al. Safety and effectiveness of immunotherapy in patients with indolent systemic mastocytosis presenting with hymenoptera venom anaphylaxis. J Allergy Clin Immunol. 2008;121:519-26.

64. Jarkvist J, Salehi C, Akin C, Gülen T. Venom immunotherapy in patients with clonal mast cell disorders: IgG4 correlates with protection. Allergy. 2020;75:169-77.
65. Bonadonna P, González de Olano D, Zanotti R, Riccio A, De Ferrari L, Lombardo C, et al. Venom immunotherapy in patients with clonal mast cell disorders: efficacy, safety, and practical considerations. J Allergy Clin Immunol Pract. 2013;1:474-8.

66. Ober AI, MacLean JA, Hannaway PJ. Life-threatening anaphylaxis to venom immunotherapy in a patient taking an angiotensin-converting enzyme inhibitor. J Allergy Clin Immunol. 2003;112:1008-9.

67. Ruëff F, Vos B, Oude Elberink J, Bender A, Chatelain R, Dugas-Breit S, et al. Predictors of clinical effectiveness of hymenoptera venom immunotherapy. Clin Exp Allergy. 2014;44:736-46.

68. Bonadonna P, Zanotti R, Pagani M, Bonifacio M, Scaffidi L, Olivieri E, et al. Anaphylactic reactions after discontinuation of hymenoptera venom immunotherapy: a clonal mast cell disorder should be suspected. J Allergy Clin Immunol Pract. 2018;6:1368-72.

69. Oude Elberink JN, de Monchy JG, Kors JW, van Doormaal JJ, Dubois AE. Fatal anaphylaxis after a yellow jacket sting, despite venom immunotherapy, in two patients with mastocytosis. JAllergy Clin Immunol. 1997;99:153-4.

70. Ruëff F, Wenderoth A, Przybilla B. Patients still reacting to a sting challenge while receiving conventional hymenoptera venom immunotherapy are protected by increased venom doses. J Allergy Clin Immunol. 2001;108:1027-32.

71. Ruëff F, Przybilla B, Müller U, Mosbech H. The sting challenge test in hymenoptera venom allergy. Position paper of theSubcommittee on Insect Venom Allergy of the European Academy of Allergology and Clinical Immunology. Allergy. 1996:51:216-25.

72. Aßmus K, Meissner M, Kaufmann R, Valesky EM. Benefits and limitations of sting challenge in hymenoptera venom allergy. Allergol Select. 2021;5:45-50.

73. Ruëff F, Przybilla B, Bilò MB, Müller U, Scheipl F, Seitz MJ, et al. Clinical effectiveness of hymenoptera venom immunotherapy: a prospective observational multicenter study of the European Academy of Allergology and Clinical Immunology interest group on insect venom hypersensitivity. PLoS One. 2013;8:e63233.

74. Lerch E, Müller UR. Long-term protection after stopping venom immunotherapy: results of re-stings in 200 patients. JAllergy Clin Immunol. 1998;101:606-12.

75. Ruiz-León B, Navas A, Serrano P, Espinazo M, LabradorHorrillo M, Monsalve RI, et al. Successful adaptation of bee venom immunotherapy in a patient monosensitized to Api m 10. J Investig Allergol Clin Immunol. 2020;30:296-8.

76. Golden DB, Kagey-Sobotka A, Valentine MD, Lichtenstein LM. Dose dependence of hymenoptera venom immunotherapy. JAllergy Clin Immunol. 1981;67:370-4.

77. Goldberg A, Confino-Cohen R. Bee venom immunotherapy-How early is it effective? Allergy. 2010;65:391-5.

78. Reisman RE. Duration of venom immunotherapy: relationship to the severity of symptoms of initial insect sting anaphylaxis. JAllergy Clin Immunol. 1993;92:831-6.

79. Golden DB, Kwiterovich KA, Kagey-Sobotka A, Valentine MD, Lichtenstein LM. Discontinuing venom immunotherapy: outcome after five years. J Allergy Clin Immunol. 1996;97:579-87.

80. Hafner T, DuBuske L, Kosnik M. Long-term efficacy of venom immunotherapy. Ann Allergy Asthma Immunol. 2008;100:162-5. 